\title{
A systematic review of the accuracy and indications for diagnostic laparoscopy prior to curative-intent resection of gastric cancer
}

\author{
Pierre-Anthony Leake • Roberta Cardoso • Rajini Seevaratnam • Laercio Lourenco • \\ Lucy Helyer · Alyson Mahar $\cdot$ Calvin Law $\cdot$ Natalie G. Coburn
}

Received: 14 February 2011/ Accepted: 17 March 2011/Published online: 11 June 2011

(c) The International Gastric Cancer Association and The Japanese Gastric Cancer Association 2011

\begin{abstract}
Background Despite improved preoperative imaging techniques, patients with incurable or unresectable gastric cancer are still subjected to non-therapeutic laparotomy. Diagnostic laparoscopy (DL) has been advocated by some to be essential in decision-making in gastric cancer. We aimed to identify and synthesize findings on the value of DL for patients with gastric cancer, in this era of improved preoperative imaging.

Methods Electronic literature searches were conducted using Medline, EMBASE, and the Cochrane Central
\end{abstract}

Electronic supplementary material The online version of this article (doi:10.1007/s10120-011-0047-z) contains supplementary material, which is available to authorized users.

\section{P.-A. Leake · C. Law · N. G. Coburn}

Department of Surgery, University of Toronto,

Toronto, ON, Canada

R. Cardoso · R. Seevaratnam - A. Mahar - N. G. Coburn Sunnybrook Research Institute, Sunnybrook Health Sciences Centre, Toronto, ON, Canada

\section{Lourenco}

Department of Surgery, Universidade Federal de Sao Paulo, Sao Paulo, Brazil

\section{Helyer}

Department of Surgery, Dalhousie University,

Halifax, NS, Canada

\section{A. Mahar}

Department of Community Health and Epidemiology,

Queen's University, Kingston, ON, Canada

\section{Law · N. G. Coburn $(\bowtie)$}

Division of Surgical Oncology, Sunnybrook Health Sciences

Centre, Odette Cancer Centre, University of Toronto,

2075 Bayview Ave, Suite T2-60, Toronto, ON, Canada

e-mail: natalie.coburn@sunnybrook.ca
Register of Controlled Trials from January 1, 1998 to December 31, 2009. We calculated the change in management and avoidance of laparotomy based on the addition of DL and laparoscopic ultrasound (LUS). The accuracy, agreement (kappa), sensitivity, and specificity of DL in assessing tumor extent, nodal involvement, and the presence of metastases with respect to the gold standard (pathology) were also calculated.

Results Twenty-one articles were included. DL showed moderate to substantial agreement with final pathology for $\mathrm{T}$ stage, but only fair agreement for $\mathrm{N}$ stage. For $\mathrm{M}$ staging, DL had an overall accuracy, sensitivity, and specificity ranging from $85-98.9 \%, 64.3-94 \%$, and $80-100 \%$, respectively. The use of DL altered treatment in $8.5-59.6 \%$ of cases, avoiding laparotomy in $8.5-43.8 \%$ of cases. LUS provided additional benefit in $5.8-7.2 \%$ of cases.

Conclusions Despite evolving preoperative imaging techniques, diagnostic laparoscopy continues to be of substantial value in staging patients with gastric cancer and in avoiding unnecessary laparotomy. The current data support DL for all patients with advanced gastric cancer.

Keywords Gastric cancer - Diagnostic laparoscopy · Laparoscopic ultrasound · Cancer staging

\section{Introduction}

Despite developments in preoperative imaging, the suboptimal identification of advanced and incurable disease, particularly carcinomatosis, results in high rates of unnecessary laparotomies in patients with gastric cancer [1-3]. Diagnostic laparoscopy has been suggested to reduce these rates by identifying patients with unrecognized incurable disease [4]. The addition of peritoneal 
lavage to diagnostic laparoscopy can identify patients with free intraperitoneal cancer cells, the presence of which connotes a poor prognosis [5]. Present guidelines recommend diagnostic laparoscopy for advanced (T3 and T4) gastric cancer without evidence of distant metastases on preoperative imaging [4]. Despite this incorporation of diagnostic laparoscopy in the gastric cancer treatment algorithm [6,7], its use remains haphazard. The uncertainty of the continued utility of diagnostic laparoscopy in the era of improved radiologic staging is likely the reason. Data for Ontario show that only $5.1 \%$ of patients undergoing curative resections had diagnostic laparoscopy [8]. In the United States, based on data from the Surveillance, Epidemiology and End Results-Medicare (SEER-Medicare) Linked Database, $8.3 \%$ of patients with gastric cancer undergoing any surgery had diagnostic laparoscopy [3].

There is great variability in the literature regarding the extent of evaluation and the organs to be inspected during diagnostic laparoscopy. The Society of Gastrointestinal and Endoscopic Surgeons (SAGES) guidelines reference the technique proposed by D'Ugo et al. [5]. A pneumoperitoneum is created and an angled laparoscope inserted via an umbilical port. Cytology is performed either on aspirated ascitic fluid or after instilling $200 \mathrm{ml}$ of normal saline in the absence of ascites [5]. Inspection of the peritoneal cavity serves three purposes: (1) evaluation of tumor depth (T stage), (2) identification of nodal disease ( $\mathrm{N}$ stage), and (3) identification of gross peritoneal or liver metastases ( $\mathrm{M}$ stage). The anterior surface of the stomach and the perigastric, porta hepatis, and gastrohepatic lymph nodes can be inspected and biopsied by mobilizing the left lateral lobe of the liver. In cases of posterior tumors, the lesser sac is opened and the tumor visualized. Laparoscopic ultrasound has been recommended to aid in the detection of deep liver metastases [4].

The goal of this systematic review is to provide a contemporary assessment of the value of diagnostic laparoscopy in gastric cancer. This review will determine the extent to which diagnostic laparoscopy benefits patients with early gastric cancer (T1 and T2), and establish whether diagnostic laparoscopy is necessary in the era of improved imaging technologies. In addition, the impact of diagnostic laparoscopy on changing the management of gastric cancer patients and avoiding unnecessary laparotomy will be assessed. Finally, the role and value of laparoscopic ultrasound will be assessed in the staging process.

\section{Methods}

Data sources

Electronic literature searches were conducted in Medline and EMBASE from January 1, 1998 to December 31, 2009 according to the search algorithm presented in Electronic Appendix A. Search terms included: [exp Stomach Cancer/ or (((gastric or stomach) adj1 cancer\$) or ((gastric or stomach) adj1 carcinoma) or ((gastric or stomach) adj1 adenocarcinoma) or ((gastric or stomach) adj1 neoplasm\$)).mp.] and [Laparoscopy/or peritoneal lavage/or laparoscopic surgery/or Laparotomy/] or [clinical trial/or controlled clinical trial/or exp comparative study/or meta analysis/or multicenter study/or exp practice guideline/or randomized controlled trial/] not [review or case report/or *gastrointestinal stromal tumor/or exp B cell lymphoma/ and "marginal zone".mp.]. A separate search of the Cochrane Central Register of Controlled Trials (1998-2009) was performed using the search term "gastric cancer". Studies were limited to English language. No attempt was made to locate unpublished material.

\section{Study selection and review process}

To be eligible, studies had to meet the following criteria: (1) studies that provided data on the role of diagnostic laparoscopy in changing management or avoiding laparotomy; (2) the correlation of laparoscopy with final pathology with respect to tumor stage, nodal status, and metastatic disease; and (3) a minimum of 30 human patients with confirmed histology of gastric adenocarcinoma. Studies were excluded according to the following exclusion criteria: (1) reviews, meta-analyses, systematic reviews, abstracts, editorials or letters, case reports, and guidelines; (2) studies where gastric adenocarcinoma data could not be extracted from pooled results; and (3) studies using animal models. No age, gender, or staging restrictions were employed. All electronic search titles, selected abstracts; and full-text articles were independently reviewed by a minimum of two reviewers $(\mathrm{NC}, \mathrm{PL}$, and LL). Reference lists from review papers and relevant articles were also examined for additional studies that met our inclusion criteria. Disagreements on study inclusion/ exclusion were resolved with a consensus meeting.

\section{Data extraction}

A systematic approach to data extraction was used to produce a descriptive summary of participants, interventions, and study findings. The first reviewer (PL) independently extracted the data and a second reviewer (RC) checked the data extraction. No attempt was made to contact authors for additional information.

Data analysis

Various definitions were found for the calculation of accuracy, sensitivity, and specificity. Therefore, values 
were re-calculated from the original numbers provided in each included publication when possible. Accuracy was defined as follows: (number of true positives + number of true negatives)/(number of true positives + false positives + true negatives + false negatives $) \times 100$. Sensitivity was calculated as follows: (number of true positives)/ (number of true positives + number of false negatives) $\times 100$. Specificity was defined as follows: (number of true negatives)/(number of true negatives + number of false positives) $\times 100$. The kappa statistic with a $95 \%$ confidence interval (CI) was calculated to obtain a quantitative measure of the magnitude of agreement between diagnostic laparoscopy and histopathology. The following interpretation of kappa was used: $<0=$ less than chance agreement, $0.01-0.20=$ slight agreement, $0.21-0.40=$ fair agreement, $0.41-0.60=$ moderate agreement, 0.61$0.80=$ substantial agreement, and $0.81-0.99=$ almost perfect agreement [9]. Descriptive characteristics including country of origin, study type, number of patients, patient characteristics, location inspected, and staging classification system used, as well as change in management and avoidance of laparotomy, were also collected for each included study.

\section{Results}

Search results

A total of 1129 abstracts/citations were identified from the electronic and hand search for preliminary review. After removal of duplicates and screening for relevant titles and abstracts, a total of 435 articles were submitted for a fulltext review. Twenty-one articles [10-30] satisfied the inclusion and exclusion criteria and were included in the review (Fig. 1). The descriptive characteristics of each included study are presented in Electronic Appendix B.

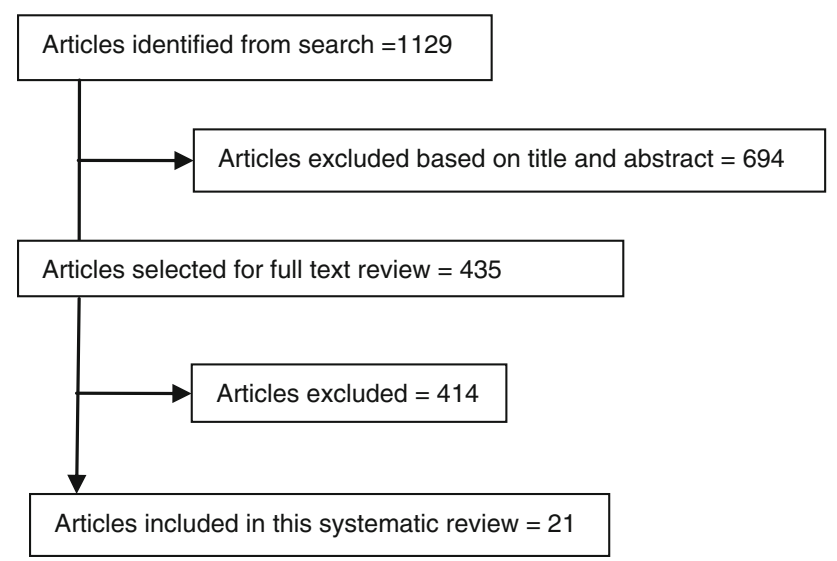

Fig. 1 Article selection flow
Study characteristics

Study design

Twelve studies were prospective $[11,13-16,18,19,22-24$, $29,30]$ and nine were retrospective $[10,12,17,20,21,25-$ 28]. Pooled data, in nine studies that included either gastric and esophagogastric cases [12, 17, 20, 23, 26, 27] or a combination of upper gastrointestinal malignancies [10, 16, 29], required data extraction specific to gastric adenocarcinoma.

\section{Tumor stage}

Four studies [11, 15, 19, 28] included patients with only advanced tumor stage (T3 and T4). Early gastric cancers (T1 and T2) were included in ten studies [14, 16-18, 20, $21,23,24,26,30]$. The tumor stage was not defined in seven studies [10, 12, 13, 22, 25, 27, 29]; however, all cases included were considered curative based on preoperative imaging.

\section{Preoperative staging}

Preoperative staging investigations included endoscopy with [10, 12, 15-18, 20, 21, 23, 26, 28] or without [14, 19, 22, 25] endoscopic ultrasound (EUS), barium studies [19], abdominal ultrasound (AUS) [10, 12, 14-19, 25, 27-30], computed tomography (CT) [10-12, 14-23, 25-28, 30], magnetic resonance imaging (MRI) [16, 18, 23, 26, 30], and positron emission tomography (PET) [18, 20]. CT was the most consistently used preoperative staging investigation, being used in 18 studies.

\section{Laparoscopic inspection}

Diagnostic laparoscopy was performed in all studies. Five studies failed to describe the procedure of laparoscopy [14, 20, 23, 26, 29]. Four studies made vague descriptions [11, 17-19]. Twelve studies specifically mentioned areas of visualization but varied by inspection criteria: peritoneal cavity [10, 12, 15, 16, 21, 22, 24, 25, 27, 28, 30], liver [10, $12,13,15,16,21,24,25,27,28,30]$, omentum [10, 12, 21, 22, 25, 27], ligamentum teres [10], spleen [10], diaphragm $[12,15,22,25,30]$, stomach $[12,13,15,22,24,25,27$, $30]$, lymph nodes $[12,24,25,30]$, pelvis [12, 13, 22, 25, 30], pancreas [15], ovaries [16], and lesser sac [13, 16, 21, 22, 30].

\section{Additional modalities}

In addition to laparoscopic inspection, peritoneal lavage or analysis of ascitic fluid was performed in nine studies [13, 
17, 19, 20, 22-24, 28, 30]. Laparoscopic ultrasound, in seven studies, was used as a supplemental method to detect advanced local, nodal, and metastatic disease [15, 16, 18, 23, 26, 27, 29].

\section{Study findings}

Study findings are summarized in Tables 1, 2, 3, and 4. The outcome measures of interest included are the accuracy, sensitivity, and specificity of diagnostic laparoscopy in predicting $\mathrm{T}$ stage, $\mathrm{N}$ stage, and the presence of metastatic disease missed by conventional preoperative staging tools. In addition, we examined the change in management and avoidance of laparotomy with diagnostic laparoscopy and laparoscopic ultrasound. The gold standard used was final pathology.

\section{Preoperative $T$ staging of gastric cancer by diagnostic laparoscopy}

Five studies assessed the use of diagnostic laparoscopy in providing an accurate $\mathrm{T}$ stage. Three of the five studies used laparoscopy alone $[11,14,21]$, while two incorporated laparoscopic ultrasound [16, 18]. Table 1 summarizes the results. The correlation between laparoscopy and final pathology for $\mathrm{T}$ stage showed moderate to substantial agreement based on kappa values ranging from 0.455 to 0.739 .

\section{Preoperative $N$ staging of gastric cancer by diagnostic} laparoscopy

Three studies [14, 16, 18] assessed the use of diagnostic laparoscopy in determining the $\mathrm{N}$ stage. As shown in Table 2, the accuracy of diagnostic laparoscopy was $64.3 \%, 66.7 \%$, and $98.9 \%$. Two of the three studies [14, 18] calculated the correlation of diagnostic laparoscopy to final pathology for $\mathrm{N}$ stage. Only fair agreement was shown between the two (kappa $=0.324-0.340)$.

\section{Preoperative $M$ staging of gastric cancer by diagnostic laparoscopy}

Table 3 summarizes the results of the fifteen studies [10, $11,14-16,18,19,21-23,25,26,28-30]$ where diagnostic

Table 1 Preoperative $\mathrm{T}$ staging of gastric cancer by diagnostic laparoscopy

\begin{tabular}{llllll}
\hline Study & Preoperative imaging used for T staging & Accuracy & Sensitivity & Specificity & Kappa (95\% CI) \\
\hline Blackshaw [11] & $\mathrm{CT}$ & $\mathrm{T} 1 / 2=90 \%^{\mathrm{b}}$ & $\mathrm{T} 1 / 2=80 \%^{\mathrm{b}}$ & $\mathrm{T} 1 / 2=91 \%^{\mathrm{b}}$ & $0.455(0.303-0.608)^{\mathrm{a}}$ \\
& & $\mathrm{T} 3=67 \%^{\mathrm{b}}$ & $\mathrm{T} 3=73 \%^{\mathrm{b}}$ & $\mathrm{T} 3=62 \%^{\mathrm{b}}$ & \\
& & $\mathrm{T} 4=77 \%^{\mathrm{b}}$ & $\mathrm{T} 4=58 \%^{\mathrm{b}}$ & $\mathrm{T} 4=93 \%^{\mathrm{b}}$ & \\
Escrig Sos [14] & $\mathrm{CT}$ & $\mathrm{OA}=84.4 \%^{\mathrm{a}}$ & $\mathrm{OA}=80.6 \%^{\mathrm{b}}$ & $\mathrm{OA}=100 \%^{\mathrm{b}}$ & $0.624(0.442-0.805)^{\mathrm{a}}$ \\
Hunerbein [16] & $\mathrm{CT} /$ EUS/MRI & $\mathrm{OA}=97.7 \%^{\mathrm{a}}$ & $\mathrm{NR}$ & $\mathrm{NR}$ & $\mathrm{NR}$ \\
Muntean [18] & $\mathrm{CT} /$ EUS/MRI & $\mathrm{OA}=92.9 \%^{\mathrm{a}}$ & $\mathrm{OA}=50 \%^{\mathrm{a}}$ & $\mathrm{OA}=100 \%^{\mathrm{a}}$ & $0.632(0.140-1.124)^{\mathrm{a}}$ \\
Onate-Ocana [21] & CT/EUS & $\mathrm{NR}$ & $\mathrm{NR}$ & $\mathrm{NR}$ & $0.739(0.590-0.887)^{\mathrm{a}}$
\end{tabular}

$K$ kappa (agreement), $C I$ confidence interval, $O A$ overall, $N R$ not reported/necessary information not provided, $C T$ computed tomography, $E U S$ endoscopic ultrasound, $M R I$ magnetic resonance imaging

${ }^{\text {a }}$ Calculated by literature review study team

b Calculations published in original manuscript

Table 2 Preoperative $\mathrm{N}$ staging of gastric cancer by diagnostic laparoscopy

\begin{tabular}{llllll}
\hline Study & Preoperative imaging used for N staging & Accuracy (OA) & Sensitivity (OA) & Specificity (OA) & Kappa (95\% CI) \\
\hline Escrig Sos [14] & CT & $66.7 \%^{\mathrm{a}}$ & $60.8 \%^{\mathrm{a}}$ & $93.8 \%^{\mathrm{b}}$ & $0.324(0.126-0.521)^{\mathrm{a}}$ \\
Hunerbein [16] & AUS/CT/EUS/MRI & $98.9 \%^{\mathrm{a}}$ & $\mathrm{NR}$ & $\mathrm{NR}$ & $\mathrm{NR}$ \\
Muntean [18] & AUS/CT/EUS/MRI/PET & $64.3 \%^{\mathrm{b}}$ & $54.5 \%^{\mathrm{b}}$ & $100 \%^{\mathrm{a}}$ & $0.340(0.011-0.668)^{\mathrm{a}}$ \\
\hline
\end{tabular}

$K$ kappa (agreement), $C I$ confidence interval, $O A$ overall, $N R$ not reported/necessary information not provided, $C T$ computed tomography, $A U S$ abdominal ultrasound, EUS endoscopic ultrasound, MRI magnetic resonance imaging, PET positron emission tomography

${ }^{a}$ Calculated by literature review study team

b Calculations published in original manuscript 
Table 3 Preoperative M staging of gastric cancer by diagnostic laparoscopy

\begin{tabular}{|c|c|c|c|c|}
\hline Study & $\begin{array}{l}\text { Preoperative imaging } \\
\text { used for } \mathrm{M} \text { staging }\end{array}$ & Accuracy & Sensitivity & Specificity \\
\hline Arnold [10] & AUS/CT & $\begin{array}{l}\text { Peritoneal }=95.9 \%^{\mathrm{a}} \\
\text { Liver }=93.9 \%^{\mathrm{a}} \\
\mathrm{OA}=89.8 \%^{\mathrm{b}}\end{array}$ & $\begin{array}{l}\text { Peritoneal }=81.8 \%^{\mathrm{a}} \\
\text { Liver }=50 \%^{\mathrm{a}} \\
\mathrm{OA}=64.3 \%^{\mathrm{b}}\end{array}$ & $\begin{array}{l}\text { Peritoneal }=100 \%^{\mathrm{a}} \\
\text { Liver }=100 \%^{\mathrm{a}} \\
\mathrm{OA}=100 \%^{\mathrm{a}}\end{array}$ \\
\hline Blackshaw [11] & CT & $\begin{array}{l}\text { Peritoneal }=85 \%^{\mathrm{b}} \\
\text { Liver }=90 \%^{\mathrm{b}} \\
\mathrm{OA}=85 \%^{\mathrm{b}}\end{array}$ & $\begin{array}{l}\text { Peritoneal }=88 \%^{\mathrm{b}} \\
\text { Liver }=79 \%^{\mathrm{b}} \\
\mathrm{OA}=94 \%^{\mathrm{b}}\end{array}$ & $\begin{array}{l}\text { Peritoneal }=83 \%^{\mathrm{b}} \\
\text { Liver }=93 \%^{\mathrm{b}} \\
\mathrm{OA}=80 \%^{\mathrm{b}}\end{array}$ \\
\hline Escrig Sos [14] & $\mathrm{CT}$ & $\begin{array}{l}\text { Peritoneal }=\mathrm{NR} \\
\text { Liver }=\mathrm{NR} \\
\mathrm{OA}=91.1 \%^{\mathrm{a}}\end{array}$ & $\begin{array}{l}\text { Peritoneal }=\mathrm{NR} \\
\text { Liver }=\mathrm{NR} \\
\mathrm{OA}=72.7 \%^{\mathrm{b}}\end{array}$ & $\begin{array}{l}\text { Peritoneal }=\mathrm{NR} \\
\text { Liver }=\mathrm{NR} \\
\mathrm{OA}=97.1 \%^{\mathrm{b}}\end{array}$ \\
\hline Feussner [15] & AUS/CT & $\begin{array}{l}\text { Peritoneal }=\mathrm{NR} \\
\text { Liver }=\mathrm{NR} \\
\mathrm{OA}=95 \%^{\mathrm{a}}\end{array}$ & $\begin{array}{l}\text { Peritoneal }=\mathrm{NR} \\
\text { Liver }=\mathrm{NR} \\
\mathrm{OA}=\mathrm{NR}\end{array}$ & $\begin{array}{l}\text { Peritoneal }=\mathrm{NR} \\
\text { Liver }=\mathrm{NR} \\
\mathrm{OA}=\mathrm{NR}\end{array}$ \\
\hline Hunerbein [16] & AUS/CT/MRI & $\begin{array}{l}\text { Peritoneal }=\mathrm{NR} \\
\text { Liver }=\mathrm{NR} \\
\mathrm{OA}=92 \%^{\mathrm{b}}\end{array}$ & $\begin{array}{l}\text { Peritoneal }=\mathrm{NR} \\
\text { Liver }=\mathrm{NR} \\
\mathrm{OA}=82 \%^{\mathrm{b}}\end{array}$ & $\begin{array}{l}\text { Peritoneal }=\mathrm{NR} \\
\text { Liver }=\mathrm{NR} \\
\mathrm{OA}=100 \%^{\mathrm{b}}\end{array}$ \\
\hline Muntean [18] & AUS/CT/MRI/PET & $\begin{array}{l}\text { Peritoneal }=\mathrm{NR} \\
\text { Liver }=\mathrm{NR} \\
\mathrm{OA}=95.5 \%^{\mathrm{b}}\end{array}$ & $\begin{array}{l}\text { Peritoneal }=\mathrm{NR} \\
\text { Liver }=\mathrm{NR} \\
\mathrm{OA}=89 \%^{\mathrm{b}}\end{array}$ & $\begin{array}{l}\text { Peritoneal }=\mathrm{NR} \\
\text { Liver }=\mathrm{NR} \\
\mathrm{OA}=100 \%^{\mathrm{b}}\end{array}$ \\
\hline Nakagawa [19] & AUS/CT & $\begin{array}{l}\text { Peritoneal }=90 \%^{\mathrm{a}} \\
\text { Liver }=\mathrm{NR} \\
\mathrm{OA}=\mathrm{NR}\end{array}$ & $\begin{array}{l}\text { Peritoneal }=73.7 \%^{\mathrm{a}} \\
\text { Liver }=\mathrm{NR} \\
\mathrm{OA}=\mathrm{NR}\end{array}$ & $\begin{array}{l}\text { Peritoneal }=100 \%^{\mathrm{a}} \\
\text { Liver }=\mathrm{NR} \\
\mathrm{OA}=\mathrm{NR}\end{array}$ \\
\hline Onate-Ocana [21] & $\mathrm{CT}$ & $\begin{array}{l}\text { Peritoneal }=98.1 \%^{\mathrm{a}} \\
\text { Liver }=\mathrm{NR} \\
\mathrm{OA}=\mathrm{NR}\end{array}$ & $\begin{array}{l}\text { Peritoneal }=98.5 \%^{\mathrm{b}} \\
\text { Liver }=\mathrm{NR} \\
\mathrm{OA}=\mathrm{NR}\end{array}$ & $\begin{array}{l}\text { Peritoneal }=97.6 \% \mathrm{~b} \\
\text { Liver }=\mathrm{NR} \\
\mathrm{OA}=\mathrm{NR}\end{array}$ \\
\hline Osorio [22] & $\mathrm{CT} / \mathrm{CXR}$ & $\begin{array}{l}\text { Peritoneal }=\mathrm{NR} \\
\text { Liver }=\mathrm{NR} \\
\mathrm{OA}=100 \%^{\mathrm{a}}\end{array}$ & $\begin{array}{l}\text { Peritoneal }=\mathrm{NR} \\
\text { Liver }=\mathrm{NR} \\
\mathrm{OA}=100 \%^{\mathrm{a}}\end{array}$ & $\begin{array}{l}\text { Peritoneal }=\mathrm{NR} \\
\text { Liver }=\mathrm{NR} \\
\mathrm{OA}=100 \%^{\mathrm{a}}\end{array}$ \\
\hline Power [23] & CT/MRI & $\begin{array}{l}\text { Peritoneal }=\mathrm{NR} \\
\text { Liver }=\mathrm{NR} \\
\mathrm{OA}=98.9 \%^{\mathrm{a}}\end{array}$ & $\begin{array}{l}\text { Peritoneal }=\mathrm{NR} \\
\text { Liver }=\mathrm{NR} \\
\mathrm{OA}=94.7 \%^{\mathrm{a}}\end{array}$ & $\begin{array}{l}\text { Peritoneal }=\mathrm{NR} \\
\text { Liver }=\mathrm{NR} \\
\mathrm{OA}=100 \%^{\mathrm{a}}\end{array}$ \\
\hline Roviaro [25] & AUS/CT & $\begin{array}{l}\text { Peritoneal }=100 \%^{\mathrm{a}} \\
\text { Liver }=\mathrm{NR} \\
\mathrm{OA}=\mathrm{NR}\end{array}$ & $\begin{array}{l}\text { Peritoneal }=100 \%^{\mathrm{a}} \\
\text { Liver }=\mathrm{NR} \\
\mathrm{OA}=\mathrm{NR}\end{array}$ & $\begin{array}{l}\text { Peritoneal }=100 \%^{\mathrm{a}} \\
\text { Liver }=\mathrm{NR} \\
\mathrm{OA}=\mathrm{NR}\end{array}$ \\
\hline Sarela [26] & MDCT/MRI & $\begin{array}{l}\text { Peritoneal }=\mathrm{NR} \\
\text { Liver }=\mathrm{NR} \\
\mathrm{OA}=91.3 \%^{\mathrm{a}}\end{array}$ & $\begin{array}{l}\text { Peritoneal }=\mathrm{NR} \\
\text { Liver }=\mathrm{NR} \\
\mathrm{OA}=72.6 \%^{\mathrm{a}}\end{array}$ & $\begin{array}{l}\text { Peritoneal }=\mathrm{NR} \\
\text { Liver }=\mathrm{NR} \\
\mathrm{OA}=100 \%^{\mathrm{a}}\end{array}$ \\
\hline Sotiropoulos [28] & AUS/CT & $\begin{array}{l}\text { Peritoneal }=100 \%^{\mathrm{a}} \\
\text { Liver }=93.3 \%^{\mathrm{a}} \\
\mathrm{OA}=91.1 \%^{\mathrm{a}}\end{array}$ & $\begin{array}{l}\text { Peritoneal }=100 \%^{\mathrm{a}} \\
\text { Liver }=0 \%^{\mathrm{a}} \\
\mathrm{OA}=71.4 \%^{\mathrm{a}}\end{array}$ & $\begin{array}{l}\text { Peritoneal }=100 \%^{\mathrm{a}} \\
\text { Liver }=100 \%^{\mathrm{a}} \\
\mathrm{OA}=100 \%^{\mathrm{a}}\end{array}$ \\
\hline Tang [29] & AUS & $\begin{array}{l}\text { Peritoneal }=96.4 \%^{\mathrm{a}} \\
\text { Liver }=\mathrm{NR} \\
\mathrm{OA}=\mathrm{NR}\end{array}$ & $\begin{array}{l}\text { Peritoneal }=87.5 \%^{\mathrm{a}} \\
\text { Liver }=\mathrm{NR} \\
\mathrm{OA}=\mathrm{NR}\end{array}$ & $\begin{array}{l}\text { Peritoneal }=100 \%^{\mathrm{a}} \\
\text { Liver }=\mathrm{NR} \\
\mathrm{OA}=\mathrm{NR}\end{array}$ \\
\hline Yano [30] & AUS/CT/MRI & $\begin{array}{l}\text { Peritoneal }=93.4 \%^{\mathrm{a}} \\
\text { Liver }=100 \%^{\mathrm{a}} \\
\mathrm{OA}=93.8 \%^{\mathrm{a}}\end{array}$ & $\begin{array}{l}\text { Peritoneal }=86.7 \%^{\mathrm{a}} \\
\text { Liver }=100 \%^{\mathrm{a}} \\
\mathrm{OA}=88.2 \%^{\mathrm{a}}\end{array}$ & $\begin{array}{l}\text { Peritoneal }=100 \%^{\mathrm{a}} \\
\text { Liver }=100 \%^{\mathrm{a}} \\
\mathrm{OA}=100 \%^{\mathrm{a}}\end{array}$ \\
\hline
\end{tabular}

$O A$ overall, $N R$ not reported/necessary information not provided, $A U S$ abdominal ultrasound, $C X R$ chest X-ray, $C T$ computed tomography, $M D C T$ multi-detector CT, MRI magnetic resonance imaging, PET positron emission tomography

${ }^{a}$ Calculated by literature review study team

${ }^{\mathrm{b}}$ Calculations published in original manuscript 
Table 4 Change in treatment strategy and avoidance of laparotomy based on diagnostic laparoscopy and laparoscopic ultrasound (LUS)

\begin{tabular}{|c|c|c|c|c|c|}
\hline Study & Complete preoperative work-up & $\begin{array}{l}\text { Change in } \\
\text { management }\end{array}$ & $\begin{array}{l}\text { Avoidance of } \\
\text { laparotomy }\end{array}$ & $\begin{array}{l}\text { Change added by } \\
\text { LUS }\end{array}$ & $\begin{array}{l}\text { Change added by } \\
\text { PL }\end{array}$ \\
\hline Arnold [10] & Endoscopy/EUS/AUS/CT (NR) & $\mathrm{OA}=18.4 \%^{\mathrm{b}}$ & $\mathrm{OA}=18.4 \%^{\mathrm{b}}$ & NA & NA \\
\hline Blackshaw [11] & $\mathrm{H}-\mathrm{CT}$ & $\mathrm{OA}=54 \%^{\mathrm{a}}$ & $\mathrm{OA}=34 \%^{\mathrm{a}}$ & NA & NA \\
\hline de Graaf [12] & Endoscopy/EUS/AUS/CT (NR) & $\mathrm{OA}=28 \%^{\mathrm{b}}$ & NA & NA & NA \\
\hline Deogracias [13] & NR & $\mathrm{OA}=12.2 \%^{\mathrm{a}}$ & $\mathrm{OA}=12.2 \%^{\mathrm{a}}$ & NA & NR \\
\hline Feussner [15] & Endoscopy/EUS/AUS/H-CT & $\mathrm{OA}=40.5 \%^{\mathrm{a}}$ & NA & $\mathrm{OA}=7.2 \%^{\mathrm{b}}$ & NR \\
\hline Hunerbein [16] & EUS/AUS/H-CT/MRI & $\mathrm{OA}=34.4 \%^{\mathrm{a}}$ & $\mathrm{OA}=23.7 \%^{\mathrm{a}}$ & NR & NA \\
\hline Kaiser [17] & Endoscopy/EUS/AUS/CT (NR) & $\mathrm{OA}=25.5 \%^{\mathrm{a}}$ & $\mathrm{OA}=25.5 \%^{\mathrm{a}}$ & NA & NA \\
\hline Muntean [18] & EUS/AUS/CT (NR)/MRI/PET & $\begin{array}{l}\mathrm{T} 1-3=33.3 \%^{\mathrm{a}} \\
\mathrm{T} 4=66.7 \%^{\mathrm{a}} \\
\mathrm{OA}=37.8 \%^{\mathrm{b}}\end{array}$ & NA & $\mathrm{OA}=6.7 \%^{\mathrm{a}}$ & NR \\
\hline Nakagawa [19] & Endoscopy/barium meal/US/CT (NR) & $\mathrm{OA}=56 \%^{\mathrm{a}}$ & $\mathrm{OA}=22 \%^{\mathrm{b}}$ & NA & NA \\
\hline Nath [20] & EUS/CT (NR)/PET & $\mathrm{OA}=28.8 \%^{\mathrm{b}}$ & $\mathrm{OA}=28.8 \%^{\mathrm{b}}$ & NA & $7.9 \%$ \\
\hline Onate-Ocana [21] & Endoscopy/EUS/CT (NR) & $\mathrm{OA}=43 \%^{\mathrm{a}}$ & $\mathrm{OA}=31.1 \%^{\mathrm{b}}$ & NA & NA \\
\hline Osorio [22] & Endoscopy/CXR/CT (NR) & $\mathrm{OA}=20.6 \%^{\mathrm{a}}$ & $\mathrm{OA}=15.9 \%^{\mathrm{a}}$ & NA & NR \\
\hline Power [23] & EUS/CT (NR)/MRI & $\begin{array}{l}\mathrm{T} 1-2=3.8 \%^{\mathrm{a}} \\
\mathrm{T} 3-4=25 \%^{\mathrm{a}} \\
\mathrm{OA}=19.1 \%^{\mathrm{b}}\end{array}$ & $\mathrm{OA}=19.1 \%^{\mathrm{b}}$ & NR & NR \\
\hline Ribeiro [24] & NR & $\mathrm{OA}=16.3 \%^{\mathrm{b}}$ & $\mathrm{OA}=16.3 \%^{\mathrm{b}}$ & NA & NR \\
\hline Roviaro [25] & Endoscopy/US/CT (NR) & $\mathrm{OA}=8.5 \%^{\mathrm{b}}$ & $\mathrm{OA}=8.5 \%^{\mathrm{b}}$ & NA & NA \\
\hline Sarela [26] & EUS/MDCT/MRI & $\mathrm{OA}=39 \%^{\mathrm{a}}$ & $\mathrm{OA}=23 \%^{\mathrm{a}}$ & NR & NA \\
\hline Smith [27] & US/H-CT & $\mathrm{OA}=59.6 \%^{\mathrm{a}}$ & $\mathrm{OA}=28.9 \%^{\mathrm{a}}$ & $\mathrm{OA}=5.8 \%$ & NA \\
\hline Sotiropoulos [28] & Endoscopy/EUS/AUS/CT (NR) & $\mathrm{OA}=31.1 \%^{\mathrm{a}}$ & $\mathrm{OA}=31.1 \%^{\mathrm{a}}$ & NA & NR \\
\hline Tang [29] & AUS & $\mathrm{OA}=25 \%$ & $\mathrm{OA}=25 \%$ & NR & NA \\
\hline Yano [30] & AUS/CT (NR)/MRI & $\mathrm{OA}=53.1 \%^{\mathrm{a}}$ & $\mathrm{OA}=43.8 \%^{\mathrm{b}}$ & NA & NR \\
\hline
\end{tabular}

LUS laparoscopic ultrasound, $P L$ peritoneal lavage, $A U S$ abdominal ultrasound, $C X R$ chest X-ray, $C T$ computed tomography, $H$-CT helical CT, MDCT multi-detector CT, EUS endoscopic ultrasound, MRI magnetic resonance imaging, PET positron emission tomography, NA not applicable, $N R$ not recorded/necessary information not provided, $O A$ overall

${ }^{\text {a }}$ Calculated by literature review study team

b Calculations published in original manuscript

laparoscopy was used to detect metastases. Eleven studies evaluated the use of diagnostic laparoscopy in visually detecting overall metastatic disease [10,11, 14-16, 18, 22, $23,26,28,30]$. Four of these studies employed laparoscopic ultrasound $[15,16,18,23]$. The accuracy of diagnostic laparoscopy ranged from $85-100 \%$. The sensitivity and specificity of the test ranged from $64.3-100 \%$ and 80-100\%, respectively. For the four studies employing laparoscopic ultrasound, the accuracy in detecting metastases was $92-98.9 \%$, compared to $85-100 \%$ in those where laparoscopic ultrasound was not used.

Eight studies specifically evaluated the role of diagnostic laparoscopy in visually detecting peritoneal metastases $[10,11,19,21,25,28-30]$. The accuracy, sensitivity, and specificity were $93.4-100 \%, 73.7-100 \%$, and $83-100 \%$, respectively. Four studies evaluated the benefit of diagnostic laparoscopy in specifically detecting liver metastases $[10,11,28,30]$. None of these studies used laparoscopic ultrasound. The accuracy, sensitivity, and specificity were $90-100 \%, 50-100 \%$, and $93-100 \%$, respectively.

\section{Change in management and avoidance of laparotomy}

Twenty studies reported the benefit of diagnostic laparoscopy in changing management based on the detection of advanced local disease or metastases [10-13, 15-30]. As shown in Table 4, diagnostic laparoscopy altered management in $8.5-59.6 \%$ of cases, with $8.5-43.8 \%$ of patients able to avoid laparotomy based on diagnostic laparoscopy. Of the studies that specifically examined change in management by stage, five studies demonstrated that diagnostic laparoscopy changed management in $25-54 \%$ of advanced (T3 and T4) cases [11, 15, 19, 23, 28], while one study [23] showed a $3.8 \%$ benefit in early gastric cancer. 


\section{The benefit of laparoscopic ultrasound}

Three studies $[15,18,27]$ commented on the additional effect of laparoscopic ultrasound on changing management. Laparoscopic ultrasound altered management in an additional $5.8-7.2 \%$ of cases.

\section{Discussion}

The use of diagnostic laparoscopy as an essential investigation in gastric cancer is not universally established. Current guidelines vary in their recommendations. SAGES [4] guidelines recommend the use of diagnostic laparoscopy in advanced (T3 and T4) cancers and are against its use in early gastric cancer (T1 and T2) (Grade B recommendation). Both the European Society for Medical Oncology (ESMO) [31] and the National Comprehensive Cancer Network (NCCN) [7] recommend the use of diagnostic laparoscopy in any patient thought to have resectable disease (Grade B recommendation). SAGES [32] also recommends that laparoscopic ultrasound be employed when staging laparoscopy is undertaken. With such variability in guidelines and expert opinion, review of the most recent data seems warranted. Additionally, with newer preoperative imaging tools, such as multi-detector CT CT (MDCT), PET, and MRI, some authors have questioned the need for diagnostic laparoscopy [26].

\section{Diagnostic laparoscopy for $\mathrm{T}$ staging}

A recent systematic review suggested that EUS, MDCT, and MRI were equivalent in establishing $\mathrm{T}$ stage in gastric cancer, but recommended EUS as the most appropriate investigative modality due to the larger body of supportive evidence [33]. Accuracy rates for EUS, MDCT, and MRI were quoted as $65-92.1 \%, 77.1-88.9 \%$, and $71.4-82.6 \%$, respectively [33]. Our review has shown moderate to substantial agreement between laparoscopic findings and final pathology, with accuracy rates of up to 97.7\%. However, the clinical relevance only seems to lie in distinguishing lesions deemed unresectable (T4) by imaging modalities from resectable (T3) ones. The use of laparoscopic ultrasound for this purpose is supported by SAGES guidelines [32]. However, a recommendation based on the included studies is not possible due to the paucity of data.

In studies where both early and advanced gastric cancer patients were included $[14,16,18]$, better accuracy was achieved when laparoscopic ultrasound was used $[16,18]$. However, although laparoscopic ultrasound appeared to be accurate in the staging of early gastric cancer, this did not impact operative management.
Diagnostic laparoscopy in early gastric cancer

The likelihood of disseminated or unresectable disease increases with $\mathrm{T}$ stage [34]. Consequently, the utility of diagnostic laparoscopy in early gastric cancer management is controversial. Our review identified only one trial [23] that specifically examined the impact of diagnostic laparoscopy on $\mathrm{T} 1$ and $\mathrm{T} 2$ cancers, showing change in management in only $3.8 \%$ of patients. In comparison, our review found the most evidence to support diagnostic laparoscopy for $\mathrm{T} 3$ and $\mathrm{T} 4$ cancers, where changes in management occurred $31.1-66.7 \%$ of the time $[10,14,17$, $18,22,28]$. Based on our findings, we would recommend diagnostic laparoscopy for patients found to have T3 and T4 cancers. Further studies need to be conducted on early gastric cancer cases, and when all $\mathrm{T}$ stages are included in an analysis, caution should be taken in interpreting the results.

The presence of metastases precluding curative resection in early gastric cancer is rare [34]. Though there may not be sufficient evidence to recommend diagnostic laparoscopy in early gastric cancer, a subset of patients may benefit - those who are misdiagnosed as having early gastric cancer. Certain prognostic factors have been identified in this subset of patients and may be used selectively to determine those who may benefit from diagnostic laparoscopy. These factors include upper-third and middlethird tumor localizations, a tumor size from 2 to $5 \mathrm{~cm}$, undifferentiated adenocarcinoma, Lauren's diffuse type, endolymphatic invasion, vascular invasion, and perineural invasion [35].

\section{Diagnostic laparoscopy for $\mathrm{N}$ staging}

We are unable to provide recommendations on the value of laparoscopy in nodal staging as our review is based on only three studies. The literature shows that conventional imaging tools lack sensitivity in identifying metastatic lymph nodes. A recent systematic review has suggested that neither AUS, EUS, MDCT, conventional MRI, nor PET can reliably confirm or exclude the presence of lymph node metastasis [2]. Further studies on the use of laparoscopy and laparoscopic ultrasound for the evaluation of nodal status would be beneficial.

\section{Diagnostic laparoscopy for M staging}

The current recommendations for preoperative staging of gastric cancer include abdominal imaging with $\mathrm{CT}$ and optional PET [7]. Based on a recent meta-analysis [36], the accuracy of CT and PET in detecting overall metastatic disease was lower than the accuracy we found in our review of diagnostic laparoscopy $(81.2 \%$ and $88.2 \%$, 
respectively, versus $89.8-100 \%$ ). In our review, up to $59.6 \%$ of patients who had been previously staged as M0 by CT, PET, or MRI were found on diagnostic laparoscopy to have incurable disease, supporting the additional benefits conferred by diagnostic laparoscopy.

Laparoscopy appears to be superior to conventional preoperative staging investigations in detecting peritoneal carcinomatosis. The accuracy of identifying peritoneal metastases by CT ranges from 30\% [37] to $100 \%$ [38], with a sensitivity as low as $28.8 \%$ [39]. The sensitivity of AUS to detect peritoneal metastases is poor, reported as 9\% [40]. PET scanning consistently shows low sensitivity in detecting peritoneal metastases, with rates of 30\% [41] and $35.3 \%$ [42].

Laparoscopy does not appear to provide additional benefit over preoperative imaging for the detection of liver metastases. Guidelines suggest the preoperative imaging modality of choice to be CT [7]. However, studies show high variability in its accuracy, sensitivity, and specificity (60-100\% [11, 38, 39, 41], 60-100\% [11, 38, 39, 41], and 91.5-100\% [11, 43], respectively). As a result, PET has been advocated as an alternative superior modality [44], although this acceptance is not universal [45]. The sensitivity rate for diagnostic laparoscopy of $0-79 \%$ found in our review is likely related to the ability of laparoscopy to only visualize superficial liver lesions. Laparoscopic ultrasound, in contrast, has the benefit of identifying deep liver metastases [32]. We are unable to comment on its benefit as an adjunct to diagnostic laparoscopy as its role for the detection of liver metastases was not examined in the included studies. The role of laparoscopic ultrasound in the identification of liver metastases requires further evaluation, but currently is recommended for this purpose by SAGES (Grade B-based on high-level, well-performed studies with varying interpretations and conclusions by expert panels) [32].

Change in management, avoidance of laparotomy, and the benefit of laparoscopic ultrasound

Based on the studies included in this systematic review, diagnostic laparoscopy changed the management of up to $59.6 \%(8.5-59.6 \%)$ of gastric cancer patients who were initially deemed resectable by preoperative imaging. This predominantly involved identifying patients who had metastatic disease and who would not benefit from either laparotomy or neoadjuvant therapy (including those who may be accrued for ongoing trials). In addition, up to $43.8 \%$ of patients who were identified as incurable avoided laparotomy altogether. This illustrates the resounding benefit of diagnostic laparoscopy as a preoperative tool for optimizing patient outcomes and preventing unnecessary physical and psychological stresses through ineffective procedures [46, 47]. The added value of laparoscopic ultrasound in changing management appears to be minimal based on the included studies. Although published data suggest that through the identification of occult metastases and involved lymph nodes, laparoscopic ultrasound improves the sensitivity of diagnostic laparoscopy, making it superior to conventional imaging [48], this was not clearly borne out in our review due to the small number of published studies.

Diagnostic laparoscopy in the era of improved preoperative imaging

With reports of improved preoperative staging of gastric cancer with advanced imaging technologies (MDCT, MRI, PET) [38, 41], it has been predicted that diagnostic laparoscopy would become obsolete. Our review does not support this premise. The one study that specified MDCT as a preoperative imaging tool [26] suggested that laparoscopy may be omitted in advanced gastric cancer patients with primary tumors not involving the gastroesophageal junction or whole stomach and who have no lymphadenopathy, as diagnostic laparoscopy did not confer any further change in management in this subset of patients. Although this subgroup was identified, there was still an overall change in management in 39\% of the patients in that study based on the addition of diagnostic laparoscopy and despite the use of MDCT preoperatively. Three of twelve studies in which the type of CT scan used for staging was not specified enrolled patients during the 2000s when advanced CT technologies were likely used [18, 20, 23]. Again, diagnostic laparoscopy resulted in management changes in $19.1-37.8 \%$ of these patients. The failure of identification of peritoneal metastases by CT, despite the use of 16- or 64-detector row scanners [1], appears to be the major contributing factor to the poor performance of CT. MRI and PET fared no better, as the studies that employed these modalities [16, 18, 20, 23, 26, 30] still showed significant changes in management based on diagnostic laparoscopy of $19.1-53.1 \%$.

\section{Conclusion}

Laparoscopy shows reasonable correlation with final pathology in identifying $\mathrm{T}$ stage, but there are insufficient data to comment on the benefit of laparoscopy in identifying lymph node involvement. Laparoscopy is additive to conventional imaging in detecting overall metastatic disease and peritoneal carcinomatosis, and therefore laparoscopy shows significant benefit in changing management and avoiding unnecessary laparotomy in patients with 
gastric cancer. Laparoscopic ultrasound has minimal additional value in this regard.

Despite evolving preoperative imaging techniques, diagnostic laparoscopy appears to sustain substantial value in the staging of patients with gastric cancer. The morbidity and mortality of non-therapeutic laparotomy can be avoided through its use. Its benefit in advanced gastric cancer is clear although the benefit of its use in early gastric cancer is questionable. Laparoscopic ultrasound does not appear to be an essential staging tool in gastric cancer.

Acknowledgments This research is funded by the Canadian Cancer Society (Grant \#019325). Dr. Coburn (Career Scientist Award) has received funding through the Ontario Ministry of Health and LongTerm Care. Dr. Law is supported by the Hanna Family Chair in Surgical Oncology.

\section{References}

1. Kim SJ, Kim HH, Kim YH, Hwang SH, Lee HS, Park do J, Kim $\mathrm{SY}$, Lee $\mathrm{KH}$, et al. Peritoneal metastasis: detection with 16- or 64-detector row CT in patients undergoing surgery for gastric cancer. Radiology. 2009;253(2):407-15.

2. Kwee RM, Kwee TC. Imaging in assessing lymph node status in gastric cancer. Gastric Cancer. 2009;12(1):6-22.

3. Karanicolas PJ, Elkin EB, Jacks LM, Strong VE, Brennan MF, Coit DG. Underuse of staging laparoscopy for gastric cancer results in a high rate of futile laparotomy in the USA. J Am Coll Surg. 2010;211(3 Suppl):S106.

4. Hori Y. Diagnostic laparoscopy guidelines: this guideline was prepared by the SAGES Guidelines Committee and reviewed and approved by the Board of Governors of the Society of American Gastrointestinal and Endoscopic Surgeons (SAGES), November 2007. Surg Endosc. 2008;22(5):1353-83.

5. D’Ugo DM, Pende V, Persiani R, Rausei S, Picciocchi A. Laparoscopic staging of gastric cancer: an overview. J Am Coll Surg. 2003;196(6):965-74.

6. Allum WH, Griffin SM, Watson A, Colin-Jones D. Guidelines for the management of oesophageal and gastric cancer. Gut. 2002;50(Suppl 5):v1-23.

7. Ajani JA, Barthel JS, Bekaii-Saab T, Bentrem DJ, D'Amico TA, Das P, Denlinger C, Fuchs CS, Gerdes H, Hayman JA, Hazard L, Hofstetter WL, Ilson DH, Keswani RN, Kleinberg LR, Korn M, Meredith K, Mulcahy MF, Orringer MB, Osarogiagbon RU, Posey JA, Sasson AR, Scott WJ, Shibata S, Strong VE, Washington MK, Willett C, Wood DE, Wright CD, Yang G. Gastric cancer. J Natl Compr Canc Netw. 2010;8(4):378-409.

8. Coburn NG, Lourenco LG, Rossi SE, Gunraj N, Mahar AL, Helyer LK, Law C, Rabeneck L, Paszat L. Management of gastric cancer in Ontario. J Surg Oncol. 2010;102(1):54-63.

9. Viera AJ, Garrett JM. Understanding interobserver agreement: the kappa statistic. Fam Med. 2005;37(5):360-3.

10. Arnold JC, Neubauer HJ, Zopf T, Schneider A, Benz C, Adamek HE, Riemann JF. Improved tumor staging by diagnostic laparoscopy. Z Gastroenterol. 1999;37(6):483-8.

11. Blackshaw GR, Barry JD, Edwards P, Allison MC, Thomas GV, Lewis WG. Laparoscopy significantly improves the perceived preoperative stage of gastric cancer. Gastric Cancer. 2003;6(4):225-9.
12. de Graaf GW, Ayantunde AA, Parsons SL, Duffy JP, Welch NT. The role of staging laparoscopy in oesophagogastric cancers. Eur J Surg Oncol. 2007;33(8):988-92.

13. Deogracias ML, Rodriguez-Sanjuan JC, de la Torre F, Garcia RA, Trugeda MS, Dominguez A, Gomez-Fleitas M. Absence of port-site metastases following staging laparoscopy for gastric carcinoma. Rev Esp Enferm Dig. 2006;98(10):755-9.

14. Escrig Sos VJ, Asencio Arana F, Todoli Tomas G, Salvador Sanchis JL, Ruiz del Castillo J, Corell Planelles R. Laparoscopic evaluation of the risk of locoregional recurrence after radical surgery for gastric cancer. Rev Esp Enferm Dig. 1999;91(6):420-32.

15. Feussner H, Omote K, Fink U, Walker SJ, Siewert JR. Pretherapeutic laparoscopic staging in advanced gastric carcinoma. Endoscopy. 1999;31(5):342-7.

16. Hunerbein M, Rau B, Hohenberger P, Schlag PM. The role of staging laparoscopy for multimodal therapy of gastrointestinal cancer. Surg Endosc. 1998;12(7):921-5.

17. Kaiser GM, Sotiropoulos GC, Fruhauf NR, Stavrou GA, Peitgen K, Pottgen C, Gerken G, Paul A, Broelsch CE. Value of staging laparoscopy for multimodal therapy planning in esophago-gastric cancer. Int Surg. 2007;92(3):128-32.

18. Muntean V, Mihailov A, Iancu C, Toganel R, Fabian O, Domsa I, Muntean MV. Staging laparoscopy in gastric cancer. Accuracy and impact on therapy. J Gastrointest Liver Dis. 2009;18(2):189-95.

19. Nakagawa S, Nashimoto A, Yabusaki H. Role of staging laparoscopy with peritoneal lavage cytology in the treatment of locally advanced gastric cancer. Gastric Cancer. 2007;10(1):29-34.

20. Nath J, Moorthy K, Taniere P, Hallissey M, Alderson D. Peritoneal lavage cytology in patients with oesophagogastric adenocarcinoma. Br J Surg. 2008;95(6):721-6.

21. Onate-Ocana LF, Gallardo-Rincon D, Aiello-Crocifoglio V, Mondragon-Sanchez R, de-la-Garza-Salazar J. The role of pretherapeutic laparoscopy in the selection of treatment for patients with gastric carcinoma: a proposal for a laparoscopic staging system. Ann Surg Oncol. 2001;8(8):624-31.

22. Osorio J, Rodriguez-Santiago J, Munoz E, Camps J, Veloso E, Marco C. Outcome of unresected gastric cancer after laparoscopic diagnosis of peritoneal carcinomatosis. Clin Transl Oncol. 2008;10(5):294-7.

23. Power DG, Schattner MA, Gerdes H, Brenner B, Markowitz AJ, Capanu M, Coit DG, Brennan M, Kelsen DP, Shah MA. Endoscopic ultrasound can improve the selection for laparoscopy in patients with localized gastric cancer. J Am Coll Surg. 2009;208(2):173-8.

24. Ribeiro U Jr, Gama-Rodrigues JJ, Safatle-Ribeiro AV, Bitelman B, Ibrahim RE, Ferreira MB, Laudanna AA, Pinotti HW. Prognostic significance of intraperitoneal free cancer cells obtained by laparoscopic peritoneal lavage in patients with gastric cancer. J Gastrointest Surg. 1998;2(3):244-9.

25. Roviaro GC, Varoli F, Sonnino D, Nucca O, Rabughino G, Scarduelli A. Can routine laparoscopy help to reduce the rate of explorative laparotomies for gastric cancer? Laparoscopy in gastric cancer. Diagn Ther Endosc. 2000;6(3):125-31.

26. Sarela AI, Lefkowitz R, Brennan MF, Karpeh MS. Selection of patients with gastric adenocarcinoma for laparoscopic staging. Am J Surg. 2006;191(1):134-8.

27. Smith A, Finch MD, John TG, Garden OJ, Brown SP. Role of laparoscopic ultrasonography in the management of patients with oesophagogastric cancer. Br J Surg. 1999;86(8):1083-7.

28. Sotiropoulos GC, Kaiser GM, Lang H, Treckmann J, Brokalaki EI, Pottgen C, Gerken G, Paul A, Broelsch CE. Staging laparoscopy in gastric cancer. Eur J Med Res. 2005;10(2):88-91.

29. Tang CN, Siu WT, Li MK. Use of diagnostic laparoscopy and laparoscopic ultrasound in the management of upper gastrointestinal malignancy. Ann Coll Surg Hong Kong. 2001;5:19-24. 
30. Yano M, Tsujinaka T, Shiozaki H, Inoue M, Sekimoto M, Doki Y, Takiguchi S, Imamura H, Taniguchi M, Monden M. Appraisal of treatment strategy by staging laparoscopy for locally advanced gastric cancer. World J Surg, 2000;24(9):1130-5 (discussion 1135-6.

31. Okines A, Verheij M, Allum W, Cunningham D, Cervantes A. Gastric cancer: ESMO Clinical Practice Guidelines for diagnosis, treatment and follow-up. Ann Oncol. 2010;21(Suppl 5):50-4.

32. Richardson W, Stefanidis D, Mittal S, Fanelli RD. SAGES guidelines for the use of laparoscopic ultrasound. Surg Endosc. 2010;24(4):745-56.

33. Kwee RM, Kwee TC, et al. Imaging in local staging of gastric cancer a systematic review. J Clin Oncol. 2007;25(15):2107-16.

34. Kumai K, Otani Y, Aiura K, Iwao Y, Sugino Y, Kubota T, Kitajima M. Distant metastasis in early gastric cancer. Gastrointest Oncol A. 1998;631.

35. Shin SH, Bae JM, Jung H, Choi MG, Lee JH, Noh JH, Sohn TS, Kim S. Clinical significance of the discrepancy between preoperative and postoperative diagnoses in gastric cancer patients. J Surg Oncol. 2010;101(5):384-8.

36. Seevaratnam R, Cardoso R, McGregor C, Lourenco LG, Mahar AL, Sutradhar R, Law C, Paszat L, Coburn NG. How useful is tumor, node, metastasis (TNM) staging of gastric cancer? A meta-analysis. Gastric Cancer. 2011.

37. D'Elia F, Zingarelli A, Palli D, Grani M, et al. Hydro-dynamic CT preoperative staging of gastric cancer correlation with pathological findings. A prospective study of 107 cases. Eur Radiol. 2000;10(12):1877-85.

38. Kim AY, Kim HJ, Ha HK. Gastric cancer by multidetector row CT: preoperative staging. Abdom Imaging. 2005;30(4):465-72.

39. Shinohara T, Ohyama S, Yamaguchi T, Muto T, Kohno A, Kato Y, Urashima M. Clinical value of multidetector row computed tomography in detecting lymph node metastasis of early gastric cancer. Eur J Surg Oncol. 2005;31(7):743-8.
40. Kayaalp C, Arda K, Orug T, Ozcay N. Value of computed tomography in addition to ultrasound for preoperative staging of gastric cancer. Eur J Surg Oncol. 2002;28(5):540-3.

41. Chen J, Cheong JH, Yun MJ, Kim J, Lim JS, Hyung WJ, Noh SH. Improvement in preoperative staging of gastric adenocarcinoma with positron emission tomography. Cancer. 2005;103(11): 2383-90.

42. Lim JS, Kim MJ, Yun MJ, Oh YT, Kim JH, Hwang HS, Park MS, Cha SW, Lee JD, Noh SH, Yoo HS, Kim KW. Comparison of CT and 18F-FDG pet for detecting peritoneal metastasis on the preoperative evaluation for gastric carcinoma. Korean J Radiol. 2006;7(4):249-56.

43. Gamon Giner R, Escrig Sos J, Salvador Sanchis JL, Ruiz del Castillo J, Garcia Vila JH, Marcote Valdivieso E. Helical CT evaluation in the preoperative staging of gastric adenocarcinoma. Rev Esp Enferm Dig. 2002;94(10):593-600.

44. Kinkel K, Lu Y, Both M, Warren RS, Thoeni RF. Detection of hepatic metastases from cancers of the gastrointestinal tract by using noninvasive imaging methods (US, CT, MR imaging, PET): a meta-analysis. Radiology. 2002;224(3):748-56.

45. Dassen AE, Lips DJ, Hoekstra CJ, Pruijt JF, Bosscha K. FDGPET has no definite role in preoperative imaging in gastric cancer. Eur J Surg Oncol. 2009;35(5):449-55.

46. Smith BR, Stabile BE. Gastric adenocarcinoma: reduction of perioperative mortality by avoidance of nontherapeutic laparotomy. J Gastrointest Surg. 2007;11(2):127-32.

47. Schwarz RE. Factors influencing change of preoperative treatment intent in a gastrointestinal cancer practice. World J Surg Oncol. 2007;5:32.

48. Rau B, Hunerbein M, Schlag PM. Is there additional information from laparoscopic ultrasound in tumor staging? Dig Surg. 2002;19(6):479-83. 